OPEN ACCESS

Edited by:

Stewart Mac Mein,

German Cancer Research Center

(DKFZ), Germany

Reviewed by:

Bouchra Tawk,

Heidelberg University Hospital,

Germany

Michael Orth

LMU Munich University Hospital,

Germany

Jason Luke Parsons,

University of Liverpool,

United Kingdom

*Correspondence:

Michael D. Story

Michael.Story@UTSouthwestern.edu

${ }^{\dagger}$ Present address: John S. Yordy,

Valley Radiation Therapy Center,

Palmer, AK, United States

${ }^{\ddagger}$ These authors share first authorship

Specialty section: This article was submitted to

Radiation Oncology,

a section of the journal

Frontiers in Oncology

Received: 10 November 2021 Accepted: 31 January 2022

Published: 25 February 2022

Citation:

Ding L, Sishc BJ, Polsdofer E, Yordy JS, Facoetti A, Ciocca M

Saha D, Pompos A, Davis AJ and Story MD (2022) Evaluation of the Response of HNSCC Cell Lines to $\gamma$ Rays and ${ }^{12} \mathrm{C}$ lons: Can Radioresistant

Tumors Be Identified and Selected for ${ }^{12} \mathrm{C}$ Ion Radiotherapy?

Front. Oncol. 12:812961.

doi: 10.3389/fonc.2022.812961

\section{Evaluation of the Response of} HNSCC Cell Lines to $\gamma$-Rays and ${ }^{12} \mathrm{C}$ Ions: Can Radioresistant Tumors Be Identified and Selected for
${ }^{12} \mathrm{C}$ Ion Radiotherapy?

\author{
Lianghao Ding ${ }^{1 \neq}$, Brock J. Sishc ${ }^{1 \neq}$, Elizabeth Polsdofer ${ }^{1 \neq}$, John S. Yordy ${ }^{1 \dagger}$, \\ Angelica Facoetti ${ }^{2}$, Mario Ciocca ${ }^{2}$, Debabrata Saha ${ }^{1}$, Arnold Pompos ${ }^{1}$, Anthony J. Davis ${ }^{1}$ \\ and Michael D. Story ${ }^{\text {* }}$

\footnotetext{
1 Univeristy of Texas Southwestern Medical Center, Department of Radiation Oncology, Dallas, TX, United States, ${ }^{2}$ Medical
} \\ Physics Unit \& Research Department, Foundazione Centro Nazionale di Adroterapia Oncologica (CNAO), Pavia, Italy
}

Head and neck squamous cell carcinoma (HNSCC) is the sixth most common malignancy worldwide. Thirty percent of patients will experience locoregional recurrence for which median survival is less than 1 year. Factors contributing to treatment failure include inherent resistance to X-rays and chemotherapy, hypoxia, epithelial to mesenchymal transition, and immune suppression. The unique properties of ${ }^{12} \mathrm{C}$ radiotherapy including enhanced cell killing, a decreased oxygen enhancement ratio, generation of complex DNA damage, and the potential to overcome immune suppression make its application well suited to the treatment of HNSCC. We examined the ${ }^{12} \mathrm{C}$ radioresponse of five HNSCC cell lines, whose surviving fraction at 3.5 Gy ranged from average to resistant when compared with a larger panel of 38 cell lines to determine if ${ }^{12} \mathrm{C}$ irradiation can overcome $\mathrm{X}$-ray radioresistance and to identify biomarkers predictive of ${ }^{12} \mathrm{C}$ radioresponse. Cells were irradiated with ${ }^{12} \mathrm{C}$ using a SOBP with an average LET of $80 \mathrm{keV} / \mu \mathrm{m}$ (CNAO: Pavia, Italy). RBE values varied depending upon endpoint used. A 37 gene signature was able to place cells in their respective radiosensitivity cohort with an accuracy of $86 \%$. Radioresistant cells were characterized by an enrichment of genes associated with radioresistance and survival mechanisms including but not limited to G2/M Checkpoint MTORC1, HIF1 $\alpha$, and PI3K/AKT/MTOR signaling. These data were used in conjunction with an in silico-based modeling approach to evaluate tumor control probability after ${ }^{12} \mathrm{C}$ irradiation that compared clinically used treatment schedules with fixed RBE values vs. the RBEs determined for each cell line. Based on the above analysis, we present the framework of a strategy to utilize biological markers to predict which HNSCC patients would benefit the most from ${ }^{12} \mathrm{C}$ radiotherapy.

Keywords: carbon ion radiotherapy, head and neck squamous cell carcinoma, radioresistance, relative biological effectiveness, prediction of radioresponse 


\section{INTRODUCTION}

The potential therapeutic advantage of particle radiotherapy was recognized in the 1940s and was based upon the physical properties of the energy deposition patterns of said particles. Since that time, particle therapy has continuously developed predominantly based upon advances in engineering, imaging, and physics. The first dedicated clinical heavy ion therapy center was opened in 1994, and this facility focused on the use of accelerated carbon ions because of the physical and biological advantages over photons and protons including steeper lateral dose penumbra at greater depths in the body, a higher LET which results in a higher relative biological effectiveness (RBE) and took into account the experience gained at the Lawrence Berkeley Laboratory where the initial results for the use of heavy charged particles as a cancer therapy took place (1-4).

Since then, carbon ion radiotherapy (CIRT) has been used against intracranial cancers, head and neck cancers, primary and metastatic lung cancers, gastrointestinal tumors, sarcomas, prostate cancer, breast cancer, and pediatric cancers at what has grown to become 12 carbon centers across the globe, although none currently exist in the USA. CIRT has been shown to exert a strong antitumor effect in tumors resistant to conventional photon therapy; however, some tumor sites have been less amenable to therapy over concerns for the response of adjacent normal tissues. Whereas, the efficacy of CIRT has been shown for nonsquamous tumors like mucosal melanomas $(5,6)$, adenocarcinomas and sarcoma $(7,8)$, and adenoid cystic carcinomas $(9,10)$; in tumors that are considered radioresistant or chemoresistant, the use of CIRT for squamous cell carcinomas, the most common type of head and neck cancer, has been limited (11-13).

Given the technologic advances in recent years for proton and heavy ion radiotherapy, the potential benefit from the increased conformity of charged particles and higher LETs seen with ${ }^{12} \mathrm{C}$ ions against head and neck squamous cell carcinoma (HNSCC) of the oral cavity, larynx, pharynx, and nasopharynx in a fashion that is beneficial for sparing organs at risk such as tissues of the oral cavity, the spinal cord, or bony structures like the mandible and vertebrae has been considered and acted upon. For example, the multi-institutional in silico trial designated ROCOCO describes the benefits of particle therapy and in particular CIRT, in a trial of reirradiation for recurrent HNSCC (14). In that study, comparing reductions in mean dose to organs at risk, particle therapy - using protons and carbon ions, both achieved reductions in complications with a dosimetric benefit for carbon ions over protons which they attributed to conformity, that is, dose to the normal tissue as suggested by other studies (15-17).

CIRT facilities have a highly limited capacity to treat the millions of individuals who are diagnosed with cancer each year, and even with new facilities coming online, it remains a limited medical resource where patients should be stratified in order to optimize the use and efficiency of CIRT. Tumors of the head and neck should be ideal for the use of CIRT because head and neck regions have functionally important anatomic sites amenable to dose conformality and the additional cell killing effects of high LET radiations, particularly for low LET-resistant (photon and proton) tumors. Furthermore, identification of patients as potentially radioresistant by omics or other analysis that requires tumor sampling, is less complicated in $\mathrm{H} \& \mathrm{~N}$ cancers based upon ease of access to tumor tissue.

Intuitively, DNA repair-related biomarkers would be particularly useful for predicting radiotherapy and chemoradiotherapy outcomes for HNSCC. Ku80, a mediator of DSB repair, was established as the first candidate DNA repair biomarker to show potential predictive value for head and neck radiotherapy in a cohort of archival HNSCC specimens from irradiated patients (18). In this series, Ku80 was overexpressed in half of tumors, and its expression was independent of all clinical and genetic covariates examined. Ku80 overexpression was an independent predictor for both locoregional failure and mortality following radiotherapy $(p<0.01)$ conferring a 9-fold greater risk of mortality at 2 years. Furthermore, using a battery of HNSCC cell lines, tumor growth and metastatic potential were determined in an orthotopic model of oral tongue cancer, including how TP53 mutations influence tumor growth and metastasis (19) and how disruptive mutations in TP53 lead to treatment failure by inhibiting radiation-induced senescence (20). Besides the identification of Ku80 and TP53 as potential negative prognostic indicator in HNSCC, Eschrich et al., using the Radiosensitivity Index (RSI) in a retrospective study of HNSCC treated with radiochemotherapy identified a radiosensitive cohort of patients that saw improved locoregional control (21). However, as argued here, identifying tumors that are likely radioresistant would seem more appropriate for the selection of patients to be treated by CIRT.

Towards that goal of defining radioresistance for patient triage, 38 HNSCC cell lines were collected and interrogated for their $\gamma$-ray radioresponse via clonogenic survival. An approximately 4-fold range of radiosensitivity as measured by SF2 or SF3.5 was determined. At the molecular level, these 38 cell lines have been assayed for basal gene and miRNA expression as well as DNA methylation, and our future goal is to integrate gene expression, miRNA expression, and methylation patterns with cell survival to characterize radioresponse.

However, for this study, these 38 HNSCC cell lines were agnostically divided into 4 groups, radiosensitive, moderately radiosensitive, moderately radioresistant and radioresistant, based on SF3.5 values. A signature of 37 genes built from the basal gene expression of each cell line was then developed that could stratify these cell lines into their respective radiosensitivity cohorts with $86 \%$ accuracy. From these 38 cell lines, five were chosen to characterize the behavior of moderately radioresistant and radioresistant cell lines to ${ }^{12} \mathrm{C}$ ion exposures to determine the radioresponse to ${ }^{12} \mathrm{C}$ ions, calculate RBE values using different endpoints, and model tumor control probabilities for a series of dose and fraction combinations to expose the variability in tumor control probability when a fixed RBE is used as opposed to a personalized RBE within a radioresistant population of HNSCC tumor cell lines.

\section{METHODS}

\section{HNSCC Cell Culture}

HNSCC cell lines were cultured in Dulbecco's modified essential media (D-MEM) supplemented with 10\% Fetal Plus brand fetal bovine serum (FBS, Atlas Biologicals, Fort Collins, CO, USA), 
penicillin/streptomycin solution (Sigma Aldrich, St. Louis, MO, USA). All cell lines were authenticated by genotyping and validated as negative for mycoplasma contamination by the Molecular Diagnostics Core Services at the Dana Farber Cancer Center, Boston, MA. All cell culture was conducted in incubators at $37^{\circ} \mathrm{C}$ in ambient $5 \% \mathrm{CO}_{2}$. See Table $\mathbf{1}$ for additional information such as the anatomical site from which the cell line was derived and other information.

\section{HNSCC Tumor Gene Expression Microarray Dataset}

An expression microarray dataset (GEO accession number GSE 67614) that was generated from 102 tumor samples collected from patients treated with a consistent protocol of surgery followed by radiotherapy based upon a prospective trial that evaluated pathologic risk features, total combined treatment duration, and postoperative radiation therapy (23) was used to evaluate the expression of genes and molecular pathways identified from the cell line gene expression data. The patient pool from which these tumors were isolated were $34 \%$ stage III and $54 \%$ stage IV, i.e., predominantly high risk, and were divided into cohorts representing those for whom their disease recurred locally/regionally (LR), had distant metastasis (DM), and who showed no evidence of disease (NED). HPV status was not determined directly; however, p16 positivity was seen in samples representing 11 patients and were split 6:5 in the recurrent setting vs. those designated as having no evidence of disease.

\section{Photon Irradiations}

Photon irradiation was conducted at the University of Texas Southwestern Medical Center using a J. L. Shepherd sealed horizontal ${ }^{137}$ Cs-sourced irradiator or at the MD Anderson Cancer Center using the "NASAtron" ${ }^{137}$ Cs irradiator. Dosimetry for these sealed source irradiators was validated on an annual basis. Briefly, for the J. L. Shepherd, irradiator cells in culture were placed on a $360^{\circ}$ platform revolving at $13 \mathrm{RPM}$, irradiated, removed from the irradiator, and immediately returned to the incubator. For the

TABLE 1 | Characteristics of HNSCC cell lines.

\begin{tabular}{|c|c|c|c|c|}
\hline Cell line & SF2 & SF3.5 & P.E. & Anatomical location \\
\hline $584 \mathrm{~A} 2$ & 0.45 & 0.119 & 0.03 & Larynx \\
\hline CAL-27 & 0.459 & 0.248 & 0.07 & Oral cavity \\
\hline FADU & 0.622 & 0.346 & 0.44 & Hypopharynx \\
\hline HN30 & 0.476 & 0.179 & 0.38 & Pharynx \\
\hline HN31 & 0.542 & 0.265 & 0.11 & LN (HN30) \\
\hline HN4 & 0.652 & 0.307 & 0.15 & REC (larynx) \\
\hline HN5 & 0.709 & 0.414 & 0.65 & REC (oral cavity) \\
\hline JHU011 & 0.447 & 0.19 & 0.1 & REC (larynx) \\
\hline JHU022 & 0.442 & 0.188 & 0.16 & LN (larynx) \\
\hline JHU029 & 0.482 & 0.196 & 0.25 & Larynx \\
\hline MDA1386LN & 0.359 & 0.117 & 0.2 & LN (MDA1386TU) \\
\hline MDA1386TU & 0.574 & 0.248 & 0.08 & Hypopharynx \\
\hline MDA686LN & 0.617 & 0.319 & 0.02 & LN (MDA686TU) \\
\hline MDA686TU & 0.624 & 0.339 & 0.08 & Oropharynx \\
\hline MDA886LN & 0.342 & 0.131 & 0.03 & LN (larynx) \\
\hline OSC19 & 0.502 & 0.241 & 0.03 & LN (oral cavity) \\
\hline $\mathrm{PCl} 13$ & 0.522 & 0.31 & 0.03 & Oral cavity \\
\hline $\mathrm{PCl}-15 \mathrm{~A}$ & 0.342 & 0.108 & 0.08 & Hypopharynx \\
\hline $\mathrm{PCl}-15 \mathrm{~B}$ & 0.392 & 0.095 & 0.13 & LN (PCl-15A) \\
\hline PJ34 & 0.507 & 0.263 & 0.14 & Oral cavity \\
\hline SCC15 & 0.456 & 0.183 & 0.07 & Oral cavity \\
\hline SCC25 & 0.529 & 0.232 & 0.09 & Oral cavity \\
\hline SCC4 & 0.667 & 0.362 & 0.24 & Oral cavity \\
\hline SCC61 & 0.74 & 0.465 & 0.64 & Oral cavity \\
\hline SCC9 & 0.73 & 0.44 & 0.28 & Oral cavity \\
\hline Sqccy1 & 0.688 & 0.345 & 0.81 & Oral cavity \\
\hline TR146 & 0.554 & 0.256 & 0.09 & REC (oral cavity) \\
\hline Tul38 & 0.575 & 0.332 & 0.12 & Oral cavity \\
\hline UMSCC1 & 0.671 & 0.358 & 0.58 & REC (oral cavity) \\
\hline UMSCC11A & 0.473 & 0.225 & 0.02 & Larynx \\
\hline UMSCC14B & 0.449 & 0.152 & 0.35 & REC (UMSCC14A) \\
\hline UMSCC17A & 0.232 & 0.056 & 0.14 & Larynx \\
\hline UMSCC17B & 0.415 & 0.168 & 0.26 & E) CT (UMSCC17A) \\
\hline UMSCC22A & 0.473 & 0.176 & 0.12 & Hypopharynx \\
\hline UMSCC22B & 0.434 & 0.113 & 0.07 & LN (UMSCC22A) \\
\hline UMSCC25 & 0.656 & 0.372 & 0.47 & LN (larynx) \\
\hline UMSCC4 $47^{a}$ & 0.259 & 0.075 & 0.09 & Oral cavity \\
\hline UMSCC4 & 0.63 & 0.342 & 0.2 & Oropharynx \\
\hline
\end{tabular}

${ }^{a} H V P$ positive. EXT, extension into adjacent tissue; REC, recurrence; LN, lymph node. Anatomical location taken from Zhao et al. (22). 
NASAtron irradiations, the source was vertically above the stage and there was no sample rotation. Both devices had dose rates of $\sim 3.25 \mathrm{~Gy} / \mathrm{min}$.

\section{${ }^{12} \mathrm{C}$ Ion Irradiations}

All ${ }^{12} \mathrm{C}$ irradiations took place at the Centro Nazionale di Adroterapia Oncologica (CNAO) facility in Pavia, Italy. Cells were irradiated in $\mathrm{T} 12.5 \mathrm{~cm}$ flasks while immersed in a water bath at $37^{\circ} \mathrm{C}$ using $\mathrm{CNAO}$ 's clinical, therapeutic quality, pencil beam scanning ${ }^{12} \mathrm{C}$-ion beam. A spread-out Bragg peak (SOBP) was created to assure a homogenous $( \pm 2.5 \%)$ physical dose. The beam quality has been previously characterized (24) and adheres to the recommendations of a NCI special panel on particle beam characterization (25). The dimensions of the SOBP were $17 \mathrm{~cm}$ in width, $7 \mathrm{~cm}$ in height, and $2 \mathrm{~cm}$ in depth. Cells were centered in the SOBP within a leucite holder with cells set back-to-back such that the depth of the cells in the upstream flask was $80 \mathrm{~mm}$ water equivalent depth (WED) while the position of cells in the downstream flask was $84 \mathrm{~mm}$ of WED. LETs at the positions where the cells were aligned were 74.1 and $89.3 \mathrm{keV} / \mu \mathrm{m}$, respectively. (No difference in biological response was seen based upon position.) The entrance LET was $16.4 \mathrm{keV} / \mu \mathrm{m}$ at a depth of $0.15 \mathrm{~mm}$. The physical dose rate was typically 0.60 Gy/min.

\section{Irradiation of Cells With a Carbon Ion SOBP}

To ensure a consistent SOBP, cells were suspended in a circulating water phantom maintained at $37^{\circ} \mathrm{C}$ in sealed T-12.5 flasks filled to the neck with complete cell culture medium containing $2 \% \mathrm{FBS}$ for a roughly 5 -min pre-exposure. This configuration provided a complete liquid/plastic interface with no ion deflection due to the presence of air. Immediately after irradiation, 2\% FBS-containing media was aspirated and replaced with $5 \mathrm{ml}$ of complete media containing $10 \%$ FBS for incubation.

\section{Clonogenic Cell Survival Assays}

Cells undergoing log phase growth at roughly $70 \%-80 \%$ maximum cell culture density were trypsinized and then seeded into T-12.5 flasks at low density in complete growth medium $8 \mathrm{~h}$ prior to irradiation. Five minutes prior to irradiation with either $\gamma$-rays or carbon ions, cell culture flasks were filled to the neck with complete growth media containing 2\% FBS. Cells were irradiated with doses of $1,2,4,6$, and 8 Gy of $\gamma$-rays, or 0.5 , $1,2,4$, and 6 Gy carbon ions. Following irradiation, growth medium containing 2\% FBS was immediately aspirated and replaced with growth medium containing 10\% FBS and dishes were allowed to incubate for $\sim 10$ population doublings based on cell-specific doubling times. The use of media with $2 \%$ FBS was simply to limit the overall volume of FBS that would be used if media containing 10\% FBS was used to completely fill the T12.5 flasks for only a few minutes as was necessary at CNAO. (See above.) Using $2 \% \mathrm{FBs}$ for such a limited time had no effect on cell growth or radioresponse.

Following incubation, cultures were rinsed with phosphatebuffered saline at a $\mathrm{pH}$ of 7.4 and fixed in a solution of $0.5 \%$ crystal violet and $10 \%$ methanol in water. After staining and drying, colonies were counted to determine the number of surviving cells following irradiation. Only colonies identified as having more than 50 cells per colony were scored as surviving, and the surviving fraction was determined by dividing the number of colonies by the product of the plating efficiency of the cell line multiplied by the number of cells seeded.

\section{Survival Curve Fits}

Survival curves were fitted based upon the Repairable Conditionally Repairable (RCR) Model as described in Equation 1 where $d$ is the dose per fraction and $a, b$, and $c$ are parameters determined using a curve fitting algorithm (26).

$$
S(d)=e^{-a d}+b d e^{-c d}
$$

The $\gamma$-ray survival assays were performed at least twice for each cell line. If the coefficient of variation at $2 \mathrm{~Gy}$ was greater than $25 \%$, they were repeated.

\section{RBE Calculations}

$\mathrm{RBE}$ values were calculated by comparing a radiosensitivity value from ${ }^{137} \mathrm{Cs}$ exposures (reference) to that same radiosensitivity value determined from ${ }^{12} \mathrm{C}$ exposures (test) as in Equation 2.

$$
\mathrm{RBE}=\frac{\text { Dose,reference }}{\text { Dose,test }}
$$

Radiosensitivity parameters included:

\section{Dose at $10 \%$ Survival}

The dose at $\mathrm{SF}_{10 \%}$ was calculated using values generated with the RCR model as described in Equation 1.

$\bar{D}_{\text {parm }}$

$\bar{D}$ was calculated using the parameters of the RCR model as shown in Equation 3 (26).

$$
\bar{D}=\frac{1}{a}+\frac{b}{c^{2}}
$$

$\bar{D}_{\text {AUC }}$

Here, $\bar{D}$ is calculated using a trapezoidal method of the area under the curve (AUC) of the survival curve assay. The trapezoidal method is similar to a Reimann sum with the exception that the area is approximated using trapezoids as opposed to rectangles.

\section{Limiting slope, $D_{0}$}

The limiting slope, $D_{0}$, represents the linear portion of the RCR fit located at the distal end of the survival curve. $D_{0}$ was calculated by plotting the linear portion of the RCR line of best fit in MATLAB and using a linear to calculate the slope. The relationship between the slope of the linear portion and $D_{0}$ is given in Equation 4.

$$
D_{0}=\frac{-1}{\text { slope }}
$$




\section{Transcriptomic Analysis of HNSCC Cell Lines and Tissues \\ Labeling and Hybridization of Microarrays}

The tumor data set was generated using Illumina Whole Genome HumanWG6 v2 arrays (GEO accession number GSE67614). The v3 Illumina Expression BeadChip was used to generate transcriptome profiles for the HNSCC cell lines. Each RNA sample with $0.5 \mu \mathrm{g}$ of total RNA was amplified using the Illumina TotalPrep RNA amplification kit with biotin UTP (Enzo) labeling. The Illumina TotalPrep RNA amplification kit uses T7 oligo(dT) primer to generate single-stranded cDNA followed by a second-strand synthesis to generate double-stranded cDNA which is then column purified. In vitro transcription was done to synthesize biotin-labeled cRNA using T7 RNA polymerase. The cRNA was column purified and then checked for size and yield using the BioRad Experion system. A total of $1.5 \mu \mathrm{g}$ of cRNA was hybridized for each array using the standard Illumina protocols with streptavidinCy3 (Amersham, Amersham, UK) being used for detection. Slides were scanned on an Illumina Beadstation. Summarized expression values for each probe sets were generated using BeadStudio 3.1 (Illumina Inc., San Diego, CA, USA).

\section{Preprocessing and Data Analysis for Gene Expression Profiling}

The Illumina BeadChip expression data were background subtracted and quantile-quantile normalized across samples using the MBCB algorithm (27-29). Normalized gene expression values were used for all the subsequent analysis. The clustering analysis was performed by calculating Euclidean distances and clustered by average method using the hclust function from the $\mathrm{R}$ base package.

\section{Classification of Radiosensitivity Groups in HNSCC Cell Lines Using Gene Expression Profiles}

The gene expression values from 38 HNSCC cell lines were ranked by $p$-values generated from an $F$-test using the $\mathrm{R}$ limma package. The top-ranked genes corresponded to those that significantly changed in more than one of the radiosensitivity groups. Feature selection was performed by incrementally expanding the gene list from 4 to 500 from the top of the gene ranking. Classification models were built using a support vector machine (SVM) algorithm and the models were validated using a 10 -fold repeated cross-validation.

\section{Molecular Pathway Analysis}

Functional pathway analysis was performed using the Gene Set Enrichment Analysis (GSEA 4.0.1) and Ingenuity Pathways Analysis (IPA) online software packages. Genes were ranked by $p$-values calculated from moderated $t$-test using the $\mathrm{R}$ limma package. The $\mathrm{R}$ limma analysis was performed to compare resistant cell lines vs. other cell lines or tumors where there was local recurrence (LR) vs. tumors where there was NED in patients treated with postoperative radiotherapy. A false-discovery rate $($ FDR $)<0.4$ was used as a cutoff for significantly enriched pathways and then interrogated against the GSEA Hallmark Pathways by Leading Edge Analysis for pathway enrichment using the metrics of $p$-value, FDR, and Normalized Enrichment Scores, whereas the IPA analysis focused on pathways were enriched based upon a Fisher's test with a $p$-value of 0.05 used as a cutoff. $Z$-scores for these pathways were then calculated with negative scores representing downregulation and positive scores representing upregulation of a given pathway.

\section{Tumor Control Probability}

The calculation of tumor control probability (TCP) is adapted from Antonovic et al. (30) and is summarized in Equation 5 where $N_{\text {vox }}$ is the number of voxels in an in silico tumor, $N_{i}$ is the number of cells in voxel $i$, and $S_{i, j}(d, L, p O 2)$ is the surviving fraction in voxel $i$ at fraction $j$ with dose $d$, oxygen partial pressure $\mathrm{pO}_{2}$, and LET $L$.

$$
\mathrm{TCP}=\exp \left\{-\sum_{i=1}^{N_{\text {vox }}} N_{i} \prod_{j=1}^{n} S_{i, j}\left(d, L, p O_{2}\right)\right\}
$$

A spherical tumor with a radius of $0.6203 \mathrm{~cm}$ (total volume 1 $\mathrm{cm}^{3}$ ) was assumed to contain $10^{8}$ cells distributed equally across the voxels in the in silico tumor simulation. The survival model utilized is the Repairable Conditionally Repairable (RCR) model which can account for changes in survival due to dose, LET, and partial oxygenation, as shown in Equation 6.

$$
\begin{aligned}
S\left(d, L, p \mathrm{O}_{2}\right)= & e^{-a(L) d / \tilde{O}\left(L, p \mathrm{O}_{2}\right)} \\
& +b(L) d / \tilde{O}\left(L, p \mathrm{O}_{2}\right) e^{-c(L) d / \tilde{O}\left(L, p \mathrm{O}_{2}\right)}
\end{aligned}
$$

As only the effect of dose on survival was accounted for Equation 5 was simplified to Equation 7 and Equation 6 was simplified to Equation 1.

$$
\mathrm{TCP}=\exp \left\{-\sum_{i=1}^{N_{\mathrm{vox}}} N_{i} \prod_{j=1}^{n} S_{i, j}(d)\right\}
$$

To take into account the effect of dose per fraction and the number of fractions on survival, Equation 1 was modified to Equation 8 where $d$ is the dose per fraction and $n$ is the number of fractions. In each simulation, the dose per fraction, $d$, is kept constant and the number of fractions, $n$, is varied to generate a TCP curve.

$$
S(d)=\left(e^{a d}+b d e^{-c d}\right)^{n}
$$

\section{RESULTS}

A collection of 38 HNSCC cell lines was tested for radioresponse via clonogenic cell survival. Surviving fraction at 2 and $3.5 \mathrm{~Gy}$ (SF2, SF3.5, respectively) was determined based upon the fitted values for SF2 and SF3.5 from the survival curves. The cell lines were clustered into four groups based upon Euclidean distances using the SF3.5 values for each cell line as depicted in Figure 1. Those groups are described as sensitive (S), moderately sensitive (MS), moderately resistant (MR), and resistant (R). The cluster is 
accompanied by the cell line identity and the respective SF3.5 values. SF3.5 was chosen based upon the conclusions of Johansen et al. (31), who suggest SF3.5 as more representative of radiosensitivity where a larger dose per fraction might be used as in stereotactic ablative radiotherapy (SAbR) or CIRT.

To determine whether there were differences in gene expression underlying these radiosensitivity cohorts, an SVM model was trained using the most significantly changed genes. As depicted in Figure 2A, a 37-gene panel could place the cell lines within their respective radiosensitivity cohort with an accuracy of $86 \%$. Figure 2 B represents the SF3.5 for the cell lines directly above in Figure $\mathbf{2 A}$ and reflects the trend in cell line radioresponse from radioresistant on the left to radiosensitive on the right. Furthermore, if cell line segregation was adjusted to identify the three radioresistant lines from all others, only 13 genes were required (Figure 2C). Genes segregating the R cohort include those associated with radio/chemoresistance (GAGE12C, GAGE2E, SPINK1), metabolic processes (PNLIPRP3), proliferation migration, invasion and metastasis (PARM1, CDH12, CYYR1, GAGE12C), and inhibition of apoptosis (SPINK1).

GSEA and IPA were used to examine differences in Hallmark Pathways (GSEA) and canonical pathway signaling (IPA). As shown in Figure 3, when the R cohort was compared with all others, cholesterol metabolism, G2M checkpoint, PI3K/AKT/ MTOR, and MTORC1 pathways were enriched with Normalized Enrichment Scores being 1.5988, 1.7422, 1.258, and 1.363, respectively. IPA pathway analysis identified HIFla and ERK/ MAPK pathways as upregulated along with a number of other pathways that were up- or downregulated based upon $Z$-scores.

These same pathways were then interrogated in a gene expression dataset derived from 102 flash-frozen HNSCC tumor specimens from patients treated by postoperative radiotherapy (PORT) using a consistent treatment protocol $(23,32,33)$. The gene expression data from 49 patients characterized as having NED and 35 patients characterized as having had LR were used. As shown in Figure 3, the GSEA

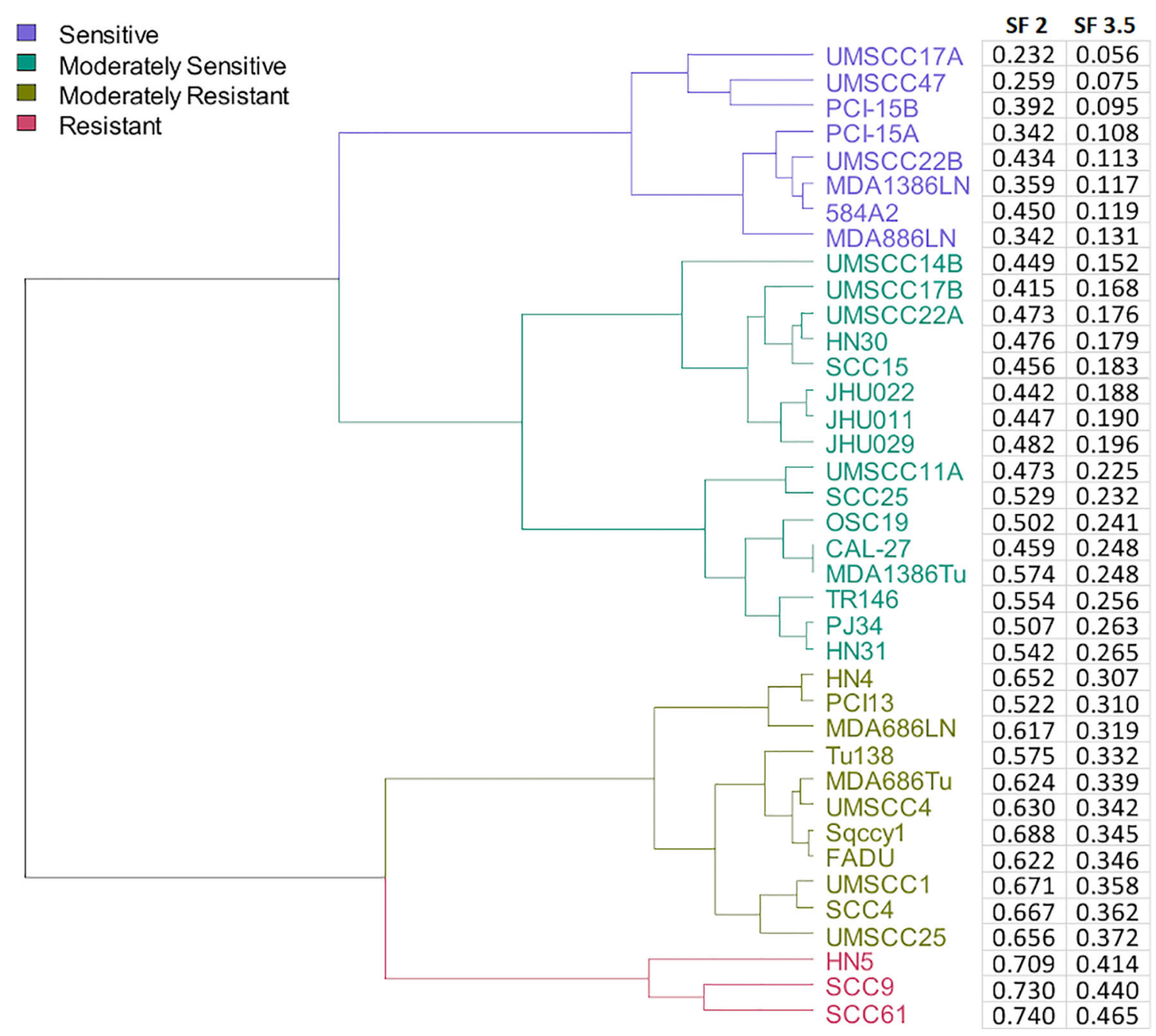

FIGURE 1 | Dendrogram of cell lines clustered by their radiosensitivity at 3.5 Gy and agnostically grouped into 4 clusters based upon radiosensitivity. 


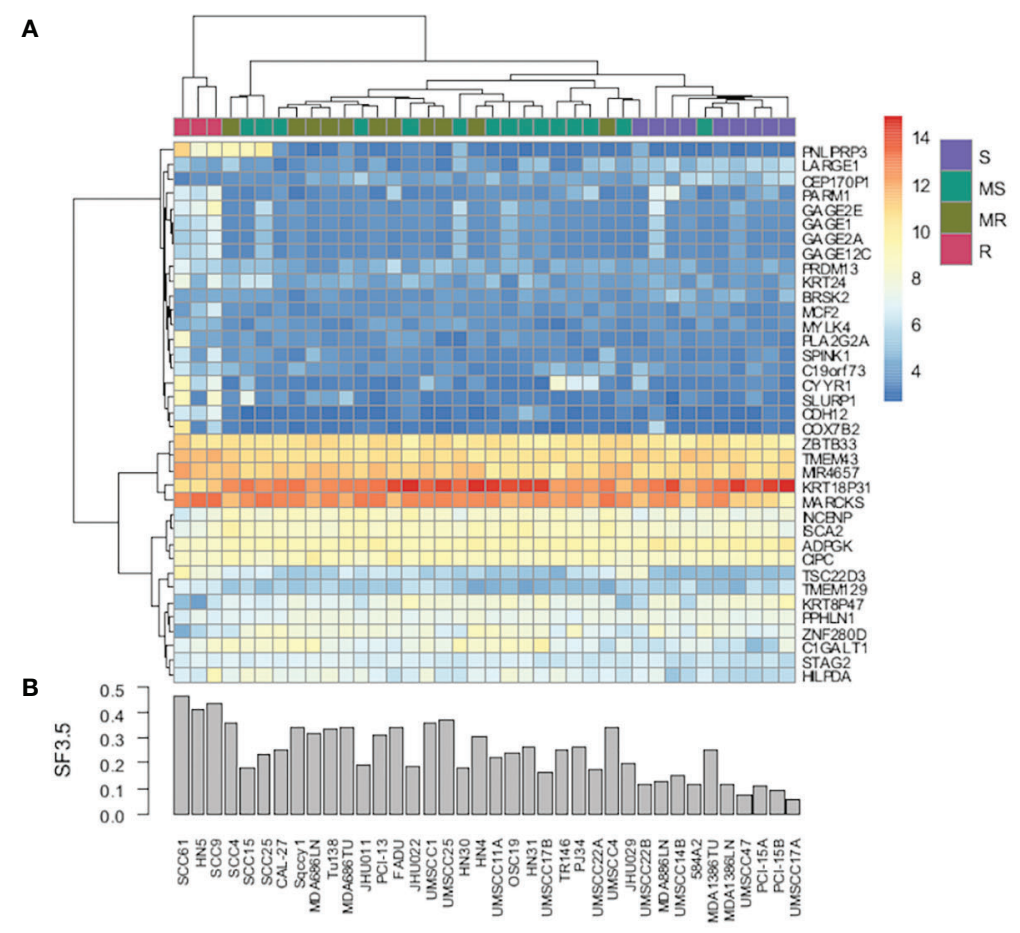

C

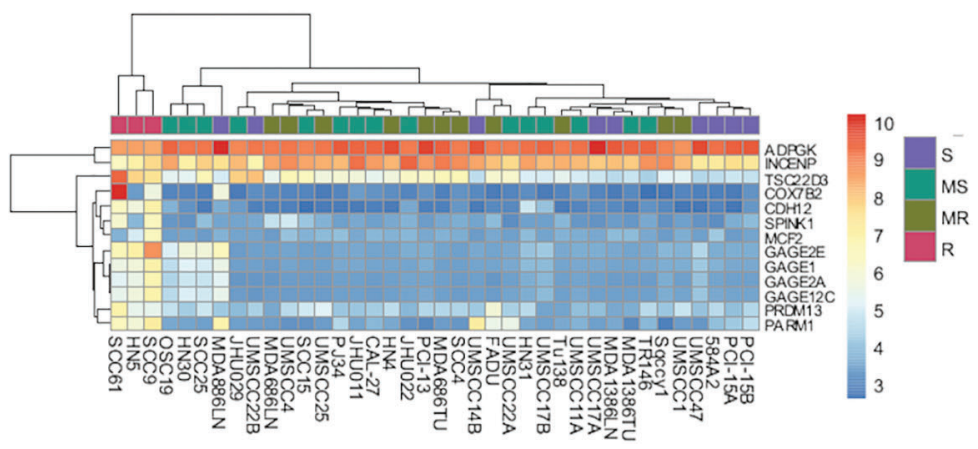

FIGURE 2 | (A) Heat map of gene expression using a 37-gene signature that segregates cell lines by radiosensitivity cluster. (B) Bar chart of radiosensitivity (SF3.5) for each cell line. (C) Heat map of gene expression using a 13-gene signature that segregates radioresistant cells from all other cell lines.

Hallmark Pathway cholesterol metabolism, G2M checkpoint, PI3K/AKT/MTOR, and MTORC1 were also enriched in the LR group when compared with those designated NED. Not surprisingly, their Normalized Enrichment Scores were somewhat lower than that seen in the cell lines.

Next, we examined the ${ }^{12} \mathrm{C}$ radioresponse of five HNSCC cell lines whose SF3.5 ranged from average to resistant when compared with the larger panel of 38 cell lines to determine if ${ }^{12} \mathrm{C}$ irradiation can overcome radioresistance. Radiation survival curves were generated using ${ }^{137} \mathrm{Cs}$ as a low LET radiation and ${ }^{12} \mathrm{C}$ ions were generated by the Carbon Therapy Center in Pavia, Italy (CNAO). The cell lines SqCC/Y1 and UMSCC1 were chosen to represent the range of SF3.5 in the MR group with an emphasis on the high end of response; HN31 represents the highest SF3.5 in the MS cohort; and HN5 and SCC9 represent the R cohort. Survival curves for these cell lines are seen in Figure 4. The RBE for ${ }^{12} \mathrm{C}$ ions was calculated at: $10 \%$ survival; by mean inactivation dose $(\bar{D})$ using the parameters of the repair-conditionally repairable curve fitting algorithm $(26) ; \bar{D}$ using a Reimann sum approach; and the limiting slopes $\left(D_{0}\right)$ for each cell line. Those values are found in Table 2 .

Using the survival data for both $\gamma$-rays and clinical ${ }^{12} \mathrm{C}$ ions from the five HNSCC cell lines, TCP curves were generated based upon Antonovic etal. (30). The calculation of TCP differs slightly from the study of Antonovic et al. (30) in that the tumor model created was fully oxygenated and the LET is fixed. Fractionation schedules (IMRT, ${ }^{12} \mathrm{C}$ ) were based upon schedules used in recurrent H\&N cancers treated at the Shanghai and Heidelberg Heavy Ion radiotherapy facilities $(34,35)$ where we chose an RBE value of 3 , which is in line with that used clinically, as well as the $\mathrm{RBE}$ values determined for each cell line. These TCP curves are depicted in Figure 5. 

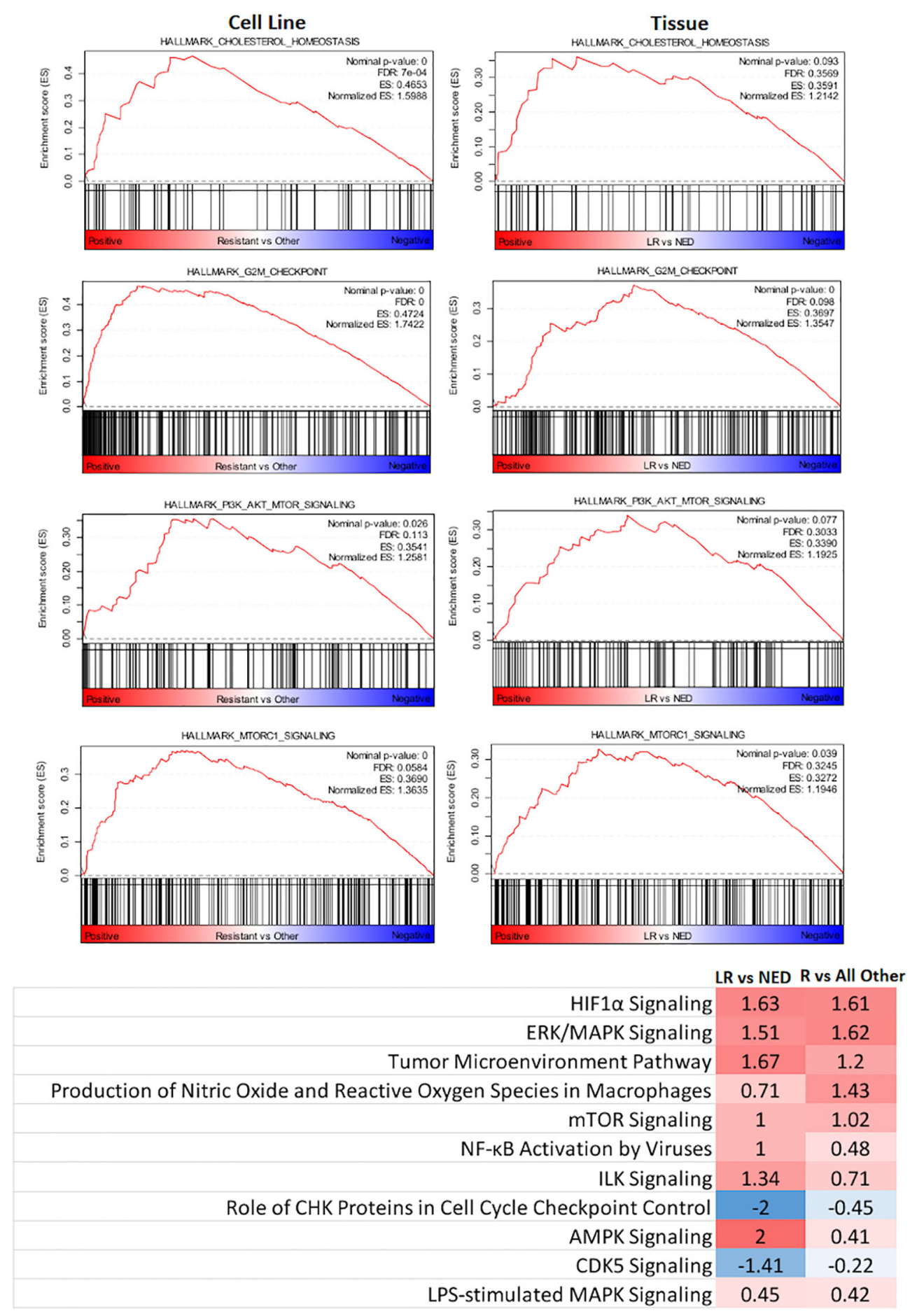

FIGURE 3 | Identification of key signaling pathways that segregate radioresistant cells from others and comparison with key signaling pathways identified in HNSCC tumors treated by PORT and classified by their treatment response: no evidence of disease (NED) and local recurrence (LR).

Lastly, in Figure 6, TCP curves representative of each cell line using the physical dose (solid line) and the GyE dose (red dashed line) are plotted, which represent the expected TCP using a generic RBE of 3. The blue dashed line in each curve represents the physical dose necessary to accomplish the same TCP as the generic RBE of 3. The differences in physical dose would represent the "underdosing" of radioresistant tumors and are found in Table 3. 


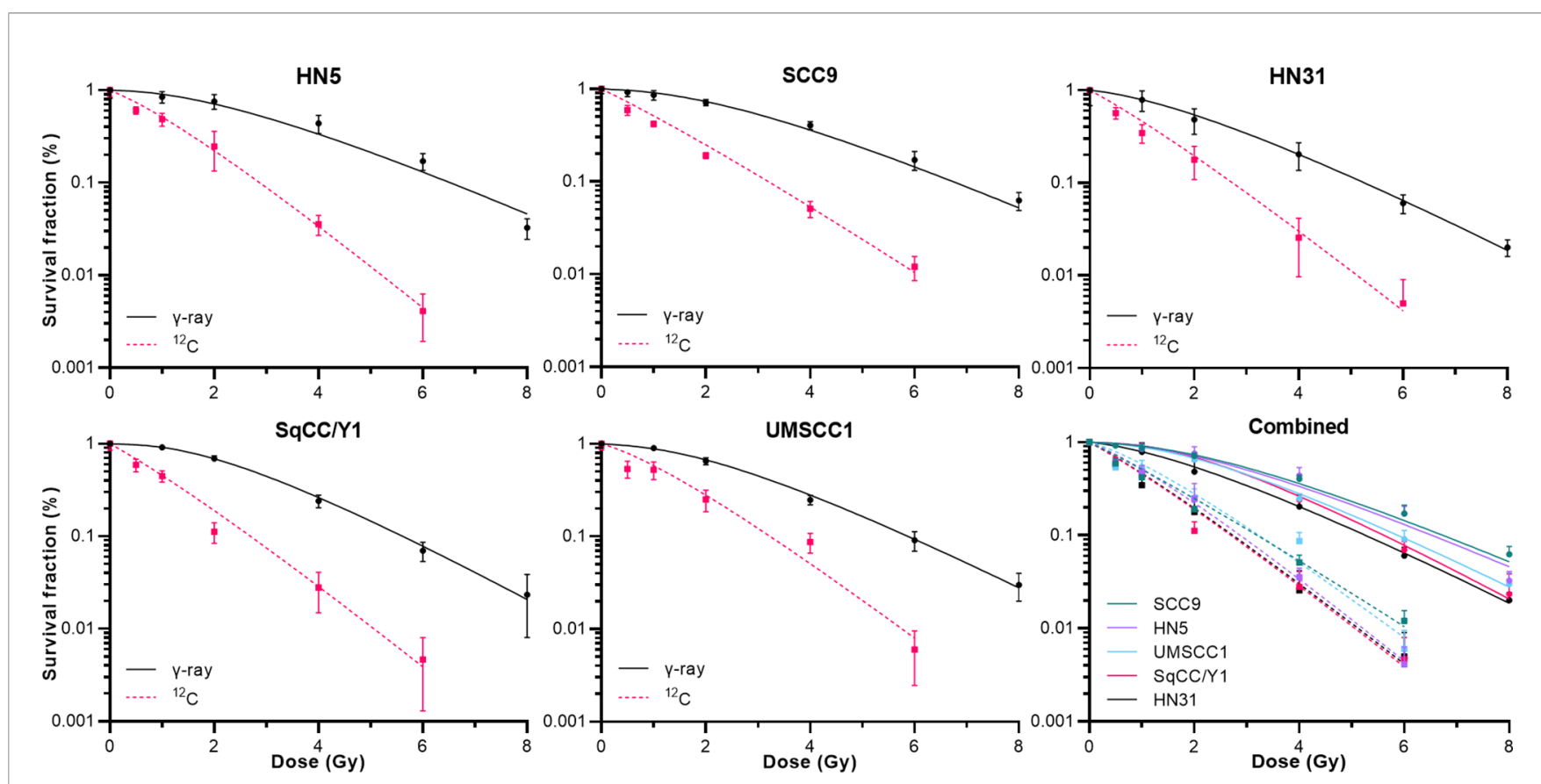

FIGURE 4 | Clonogenic cell survival for the 5 cell lines described in Table 2 when irradiated by either $\gamma$-rays or ${ }^{12} \mathrm{C}$ at 400 Mev/u. The lower right panel shows all data combined.

TABLE 2 | Limiting slopes, mean inactivation doses, and RBEs using different methods of determination.

\begin{tabular}{|c|c|c|c|c|}
\hline Cell line & RBE $_{S F 10 \%}$ & RBED $\overline{\mathbf{D}}_{\text {parm }}$ & RBED $\bar{D}_{\text {AUC }}$ & RBE $_{\mathrm{DO}}$ \\
\hline scc9 & 2.11 & 2.55 & 2.55 & 1.51 \\
\hline HN5 & 2.27 & 2.61 & 2.56 & 1.93 \\
\hline UMSCC1 & 1.83 & 2.09 & 2.07 & 1.50 \\
\hline $\mathrm{SqCC} / \mathrm{Y} 1$ & 2.08 & 2.58 & 2.57 & 1.51 \\
\hline HN31 & 1.92 & 2.14 & 2.12 & 1.61 \\
\hline Average & 2.04 & 2.39 & 2.37 & 1.61 \\
\hline Std dev & 0.17 & 0.26 & 0.25 & 0.18 \\
\hline CV & 0.08 & 0.11 & 0.11 & 0.11 \\
\hline
\end{tabular}

$R B E_{S F 10 \%}, R B E$ calculated using $10 \%$ survival; $R B E \bar{D}_{\text {parm }}, R B E$ calculated using mean inactivation dose derived from $R C R$ parameters; $R B E \bar{D}_{A U C}, R B E$ calculated using mean inactivation dose derived from Reimann sum; $R B E_{D O}, R B E$ calculated as ratio of limiting slopes.

\section{DISCUSSION}

HNSCC is the sixth most prevalent cancer in the world, and despite many treatment options, local or regional recurrence is still as high as $30 \%-50 \%$ after surgery or surgery plus radiochemotherapy. The alternatives for recurrent HNSCC include surgery with or without adjuvant radiochemotherapy with survival rates of $40 \%-66 \%$ (36), but the rates of grade 3 or grade 4 toxicities can be as high as $40 \%$ (37-41) with only 1 in 3 patients surviving reirradiation without recurrence or severe complications (42).

The high total doses from prior radiotherapy limit the total dose acceptable for reirradiation based upon the toxicity to organs at risk. However, a retrospective analysis of patients treated at the Heidelberg Ion-beam Therapy Center (HIT) by reirradiation for recurrent HNSCC with CIRT developed little in the way of either acute or late severe ( $>$ Grade III) toxicity (35) using a median total dose of $51 \mathrm{GyE}$ in $3 \mathrm{GyE}$ fractions. The Shanghai Proton and Heavy
Ion Center in a trial of recurrent HNSCC found similar results (34). Late toxicities (> Grade III) were seen in $7.1 \%$ of patients as compared to HIT where late toxicities occurred in $14.5 \%$ of patients. The primary tumor sites in these two series of patients were vastly different with Shanghai for instance having $83 \%$ of cases represented by squamous carcinomas (nasopharyngeal cancer representative of $78 \%$ of all cases) while only $28 \%$ of the HIT cohort was squamous carcinoma. These differences in patient population likely drive the differences in toxicity profiles and compare well to reirradiation trials for head and neck cancers using proton therapy where late toxicities ranged from $7 \%$ to $8 \%, 20 \%$, and $24.6 \%$, respectively (43-45).

The comparable overall improvements in late normal tissue toxicities in $\mathrm{H} \& \mathrm{~N}$ cancers treated with CIRT suggest the potential for overall improvements in outcome in primary and recurrent disease as well as the potential for dose escalation in tumors identified as radioresistant. Also, given the limitations of access, 

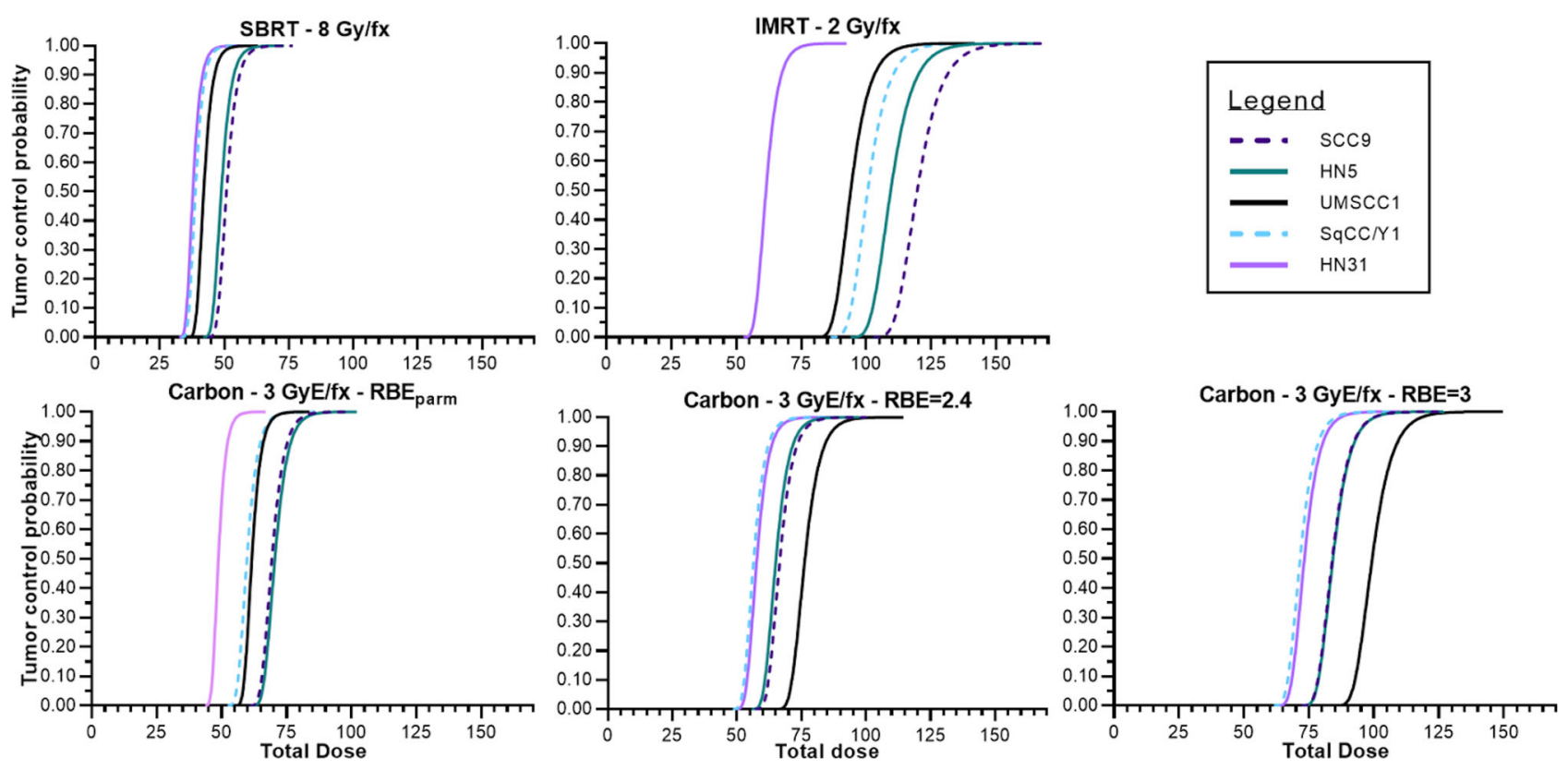

FIGURE 5 | Tumor control probabilities for various treatment modalities across the panel of HNSCC cell lines assuming no hypoxia, tumors that are a volume of $1 \mathrm{~cm}$ and containing $10^{8}$ tumor cells. Photon IMRT, SAbR, ${ }^{12} \mathrm{C}$ using a fixed RBE to determine GyE, ${ }^{12} \mathrm{C}$ using the RBE associated with the cell line used for tumor modeling.

if patients with tumors that are radioresistant can be identified, the argument could be made to treat those patients where possible with ${ }^{12} \mathrm{C}$ ions. Radioresistant HNSCC can be manifested via a number of mechanisms, the predominant mechanism being hypoxia, but resistance may also be through acquired mechanisms of enhanced DNA repair, abrogation of apoptosis or through the development of a tolerogenic immune environment. ${ }^{12} \mathrm{C}$ ions-or for that matter other ions separately or in combination-should at least partially overcome these limitations.

Determining radioresistance in a clinically useful manner is not straightforward as there are currently no clinically useful biomarkers of radioresistance in HNSCC with the possible exception of HPV status. Because of the ease with which tumors can be biopsied in H\&N cancers, an omics approach is feasible. To test this approach, 38 HNSCC tumor cell lines were tested for radiosensitivity via clonogenic survival. Basal gene expression analysis was then tested as a surrogate for clonogenic survival and found to be capable of segregating the most radioresistant cell lines from the other cell lines. Furthermore, the gene expression patterns in these radioresistant cell lines supported their classification as radioresistant. Besides identifying some of the more commonly identified genes and pathways associated with radioresistance, one of the more intriguing finds is the increased expression of the GAGE genes. These genes are not expressed in normal tissue with the exception of testes. In tumors, members of this family (GAGE1 and 2) are CD4+T cell antigens, attracting $\mathrm{T}$ cells into tumors. GAGE12 family members have been shown to increase both radio- and chemoresistance and metastasis (46-48). Other genes identified included SPINK1, an inhibitor of apoptosis that has been associated with chemoresistance (49, 50), and PARM1 which is an androgen-related gene that drives tumor proliferation $(51,52)$. Mechanistic analysis of any role for the genes in HNSCC radioresistance is warranted.

Assuming that one will be able to identify radioresistance so that patients could be triaged to receive ${ }^{12} \mathrm{C}$ ion therapy is of little benefit if this cohort of patients is then not treated to full potency. That potency is based upon an understanding of the dose equivalence for a given situation-or individual. Unlike stochastic processes such as radiation-induced carcinogenesis which uses the Seivert (Sv) for dose equivalent, dose equivalent for ${ }^{12} \mathrm{C}$ radiotherapy is described as GyEq, GyE, or GyRBE because there is no unit definition for dose equivalent in a deterministic setting. GyEq relies on the determination of the $\mathrm{RBE}$ for a given endpoint. The earliest determinations of RBE, still used today, were based upon cell survival in mostly rodent $(\mathrm{CHO})$ cell lines and the human radioresistant salivary tumor cell line HSG, which was determined to be contaminated with HeLa cells $(53,54)$. The initial ${ }^{12} \mathrm{C}$ scattered beam at NIRS was normalized to the dose-averaged LET at $80 \mathrm{KeV} / \mu \mathrm{m}$ using their clinical neutron $\mathrm{RBE}$ of 3.0 as they were equally effective (55). This along with their experience with neutron exposures led them to use this value in their NIRS treatment planning system across all tumor sites. However, it is likely that "clinical RBE" is not a fixed value and should be personalized where possible. Furthermore, RBE can be a poor descriptor of the radiobiology associated with charged particle therapy as there are many factors that determine a RBE value, and it suffers from the inability to make direct comparisons from one method of determination to another or treatment regime to another $(26,30,56)$. Therefore, we determined the 
TABLE 3 | Comparisons of GyE based upon use of a fixed RBE vs. the actual RBE for a given cell line and the dose differential for a tumor control probability of $70 \%$.

\begin{tabular}{|c|c|c|c|c|c|}
\hline Cell line & Dose (GyE) if RBE = 3 & Physical dose (Gy) & Dose (GyE) if actual RBE used & RBE (actual) & Difference in total GyE (fixed RBE vs. actual) \\
\hline scc9 & 86.9 & 28.9 & 73.9 & 2.55 & 13 \\
\hline HN5 & 87.2 & 29 & 75.9 & 2.61 & 11.3 \\
\hline UMSCC1 & 102.8 & 34.2 & 71.5 & 2.09 & 31.3 \\
\hline $\mathrm{SqCC} / \mathrm{Y} 1$ & 74.1 & 24.7 & 63.7 & 2.58 & 10.4 \\
\hline HN31 & 75.8 & 25.2 & 54 & 2.14 & 21.8 \\
\hline
\end{tabular}

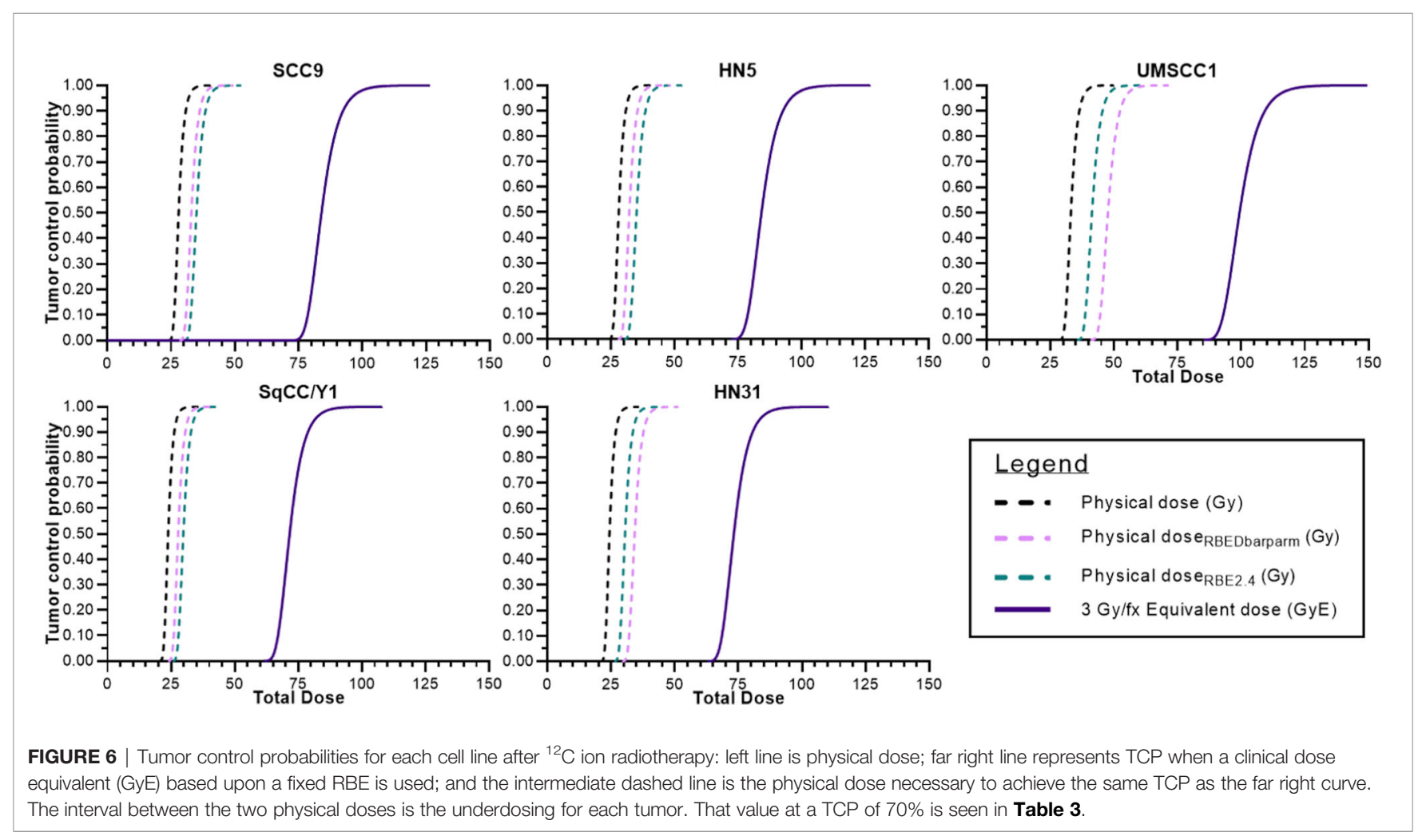

${ }^{12} \mathrm{C}$ RBE in a small cohort of moderate to radioresistant cell lines using multiple endpoints and then applied the survival and RBE values to model tumor control probability and asked whether individualization of RBE could be consequential when compared to a generic value.

Figure 5 depicts tumor control probability curves for the 5 tumor cell lines. Dose fractionation schemes include a standard 2 Gy/fraction scheme, a SAbR fractionation scheme of 8 Gy per fraction, and a $3 \mathrm{GyE}$ per fraction approach (generic RBE of 3 ). As expected, TCP varied according to the radiosensitivity of the tumor with the most resistant tumors requiring greater overall total doses to achieve the modeled tumor cure. The TCP curves for the HN31, which is intermediate in radiosensitivity, is far to the left of the more radioresistant lines in the upper left panel (IMRT 2 Gy/fraction) and at 60-70 Gy total dose TCP is roughly between $30 \%$ and $90 \%$. The remaining TCP curves for the more radioresistant cells falls well outside with total doses of 90-120 Gy required to achieve 50\% control. Total doses for the TCP of a SAbR regimen are far less but also suffer from a lack of efficacy for very radioresistant tumors and would be expected to be constrained by an increased risk for normal tissue complications.
The TCP curves for ${ }^{12} \mathrm{C}$, because this is $\mathrm{GyE}$ and uses a more conventional fraction size, is comparable with the conventional $2 \mathrm{~Gy} /$ fraction regimen. The SabR approach with ${ }^{12} \mathrm{C}$ ions may be more effective for carbon ions but suffers the same complications as a conventional SAbR approach within the radioresistant subset.

In Figure 6, we attempted to determine the underdosing for a given tumor if a fixed RBE vs. an individually determined RBE was used. TCP curves include (a) the TCP using the generic RBE of 3 for each cell line from Figure 5; (b) the 12C physical dose associated with the TCP determined using the generic RBE; and (c) the physical dose if the TCP curve based upon an RBE of 3 were modified by the RBE determined for each cell line. That difference in the physical dose curves could be considered as an "underdosing" of ${ }^{12} \mathrm{C}$ physical dose and it is consequential in that it reflects the need to understand the intrinsic radioresistance for a given tumor. Those values of "underdosing" at a TCP of 70\% are given in Table 3.

In this manuscript, we have attempted to draw attention to the need for individualizing therapy based upon the intrinsic radioresponse of a tumor. Head and neck squamous cells were used because of the recent trials where HNSCC are being treated 
with ${ }^{12} \mathrm{C}$ and because HNSCC outcomes have seen little improvement. At least for cell lines, gene expression was able to segregate radioresistant tumor cell lines from cell lines less resistant. One could argue that 38 cell lines do not bring enough diversity of radiosensitivity as the most radioresistant cell lines were few in number. Also, while there was overlap in the enrichment of specific pathways in common between cell lines and tumors that were treated with PORT, it is evident that much work is needed, irrespective of the gene expression analysis.

Using five cell lines whose radiosensitivity ranged from moderate to resistant across the 38 cell line panel, the potential impact of intrinsic radiosensitivity was tested by determining TCP curves using various radiation regimens for both $\gamma$-ray and ${ }^{12} \mathrm{C}$ exposures. This exercise highlighted the potential for underdosing radioresistant tumors when generic rather than personalized RBEs were used, which would negate the impact of triaging patients with radioresistant tumors.

We understand that our TCP calculations reflect fully oxygenated tumors, did not reflect tumor response to varied LETs, etc., but our goal is more one of relative comparisons and not necessarily quantitative comparisons. What we require are data from actual tumors treated with curative intent using $\mathrm{X}$-rays and ${ }^{12} \mathrm{C}$ ions with different dose and fractionation schedules and a realistic understanding of the impact of tumor hypoxia to challenge and ultimately improve upon the biophysical modeling of CIRT with the intent to ultimately personalize therapy.

\section{DATA AVAILABILITY STATEMENT}

The original contributions presented in the study are included in the article/supplementary materials. Further inquiries can be directed to the corresponding author.

\section{REFERENCES}

1. Castro JR, Quivey JM, Lyman JT, Chen GT, Phillips TL, Tobias CA. Radiotherapy With Heavy Charged Particles at Lawrence Berkeley Laboratory. J Can Assoc Radiol (1980) 31:30-4.

2. Blakely EA, Faddegon B, Tinkle C, Bloch C, Dominello M, Griffin RJ, et al. Three Discipline Collaborative Radiation Therapy (3DCRT) Special Debate: The United States Needs at Least One Carbon Ion Facility. J Appl Clin Med Phys (2019) 20:6-13. doi: 10.1002/acm2.12727

3. Malouff TD, Mahajan A, Krishnan S, Beltran C, Seneviratne DS, Trifiletti DM. Carbon Ion Therapy: A Modern Review of an Emerging Technology. Front Oncol (2020) 10:82. doi: 10.3389/fonc.2020.00082

4. Tinganelli W, Durante M. Carbon Ion Radiobiology. Cancers (Basel) (2020) 12. doi: $10.3390 /$ cancers 12103022

5. Yanagi T, Mizoe JE, Hasegawa A, Takagi R, Bessho H, Onda T, et al. Mucosal Malignant Melanoma of the Head and Neck Treated by Carbon Ion Radiotherapy. Int J Radiat Oncol Biol Phys (2009) 74:15-20. doi: 10.1016/ j.ijrobp.2008.07.056

6. Demizu Y, Fujii O, Terashima K, Mima M, Hashimoto N, Niwa Y, et al. Particle Therapy for Mucosal Melanoma of the Head and Neck. A SingleInstitution Retrospective Comparison of Proton and Carbon Ion Therapy. Strahlenther Onkol (2014) 190:186-91. doi: 10.1007/s00066-013-0489-9

7. Jingu K, Tsujii H, Mizoe JE, Hasegawa A, Bessho H, Takagi R, et al. Carbon Ion Radiation Therapy Improves the Prognosis of Unresectable Adult Bone and Soft-Tissue Sarcoma of the Head and Neck. Int J Radiat Oncol Biol Phys (2012) 82:2125-31. doi: 10.1016/j.ijrobp.2010.08.043

\section{AUTHOR CONTRIBUTIONS}

MS conceived of and carried out experiments, wrote the manuscript, and led the research program. $\mathrm{AD}$ conceived of and carried out experiments, edited the manuscript, and participated intellectually. AP was responsible for radiation physics, helped develop the beam configuration, and wrote a portion of the manuscript. DS was partly responsible for designing and carrying out experiments. MC was responsible for $12 \mathrm{C}$ dosimetry, developing the SOBP for $\mathrm{CNAO}$, and all physics aspects of $12 \mathrm{C}$ irradiation. AF was the CNAO biology contact person. She helped facilitate experiments and helped with the design of our irradiation setup at CNAO. JY was responsible for collecting the panel of $\mathrm{H} \& \mathrm{~N}$ tumor cell lines, designed and carried out experiments to determine the X-ray survival parameters for these cells, and provided DNA and RNA for omics analysis. EP carried out additional survival X-ray survival curves, plotted all data, and calculated survival fits and tumor control probability curves. BS conceived of and carried out both X-ray and 12C experiments. He was involved in the interpretation of data and wrote sections of this manuscript. LD was responsible for all omics analysis, interpretation of that data, and wrote sections of the manuscript. All authors listed have made a substantial, direct, and intellectual contribution to the work and approved it for publication.

\section{FUNDING}

Funds were provided through the Department of Radiation Oncology Seed Grant Program to MS as well as the David A. Pistenmaa M.D., Ph.D. Distinguished Chair in Radiation Oncology to MS. The funders were not involved in the study design, collection, analysis, interpretation of data, the writing of this article or the decision to submit it for publication.
8. Koto M, Hasegawa A, Takagi R, Sasahara G, Ikawa H, Mizoe JE, et al. Feasibility of Carbon Ion Radiotherapy for Locally Advanced Sinonasal Adenocarcinoma. Radiother Oncol (2014) 113:60-5. doi: 10.1016/ j.radonc.2014.09.009

9. Schulz-Ertner D, Didinger B, Nikoghosyan A, Jakel O, Zuna I, Wannenmacher M, et al. Optimization of Radiation Therapy for Locally Advanced Adenoid Cystic Carcinomas With Infiltration of the Skull Base Using Photon IntensityModulated Radiation Therapy (IMRT) and a Carbon Ion Boost. Strahlenther Onkol (2003) 179:345-51. doi: 10.1007/s00066-003-1071-7

10. Takagi M, Demizu Y, Hashimoto N, Mima M, Terashima K, Fujii O, et al. Treatment Outcomes of Particle Radiotherapy Using Protons or Carbon Ions as a Single-Modality Therapy for Adenoid Cystic Carcinoma of the Head and Neck. Radiother Oncol (2014) 113:364-70. doi: 10.1016/j.radonc.2014.11.031

11. Mizoe JE, Tsujii H, Kamada T, Matsuoka Y, Tsuji H, Osaka Y, et al. Dose Escalation Study of Carbon Ion Radiotherapy for Locally Advanced Headand-Neck Cancer. Int J Radiat Oncol Biol Phys (2004) 60:358-64. doi: 10.1016/ j.ijrobp.2004.02.067

12. Mizoe JE, Hasegawa A, Jingu K, Takagi R, Bessyo H, Morikawa T, et al. Results of Carbon Ion Radiotherapy for Head and Neck Cancer. Radiother Oncol (2012) 103:32-7. doi: 10.1016/j.radonc.2011.12.013

13. Morimoto K, Demizu Y, Hashimoto N, Mima M, Terashima K, Fujii O, et al. Particle Radiotherapy Using Protons or Carbon Ions for Unresectable Locally Advanced Head and Neck Cancers With Skull Base Invasion. Jpn J Clin Oncol (2014) 44:428-34. doi: 10.1093/jjco/hyu010

14. Eekers DBP, Roelofs E, Jelen U, Kirk M, Granzier M, Ammazzalorso F, et al. Benefit of Particle Therapy in Re-Irradiation of Head and Neck Patients. 
Results of a Multicentric in Silico ROCOCO Trial. Radiother Oncol (2016) 121:387-94. doi: 10.1016/j.radonc.2016.08.020

15. Weber U, Kraft G. Comparison of Carbon Ions Versus Protons. Cancer J (2009) 15:325-32. doi: 10.1097/PPO.0b013e3181b01935

16. Grun R, Friedrich T, Kramer M, Zink K, Durante M, Engenhart-Cabillic R, et al. Assessment of Potential Advantages of Relevant Ions for Particle Therapy: A Model Based Study. Med Phys (2015) 42:1037-47. doi: 10.1118/ 1.4905374

17. Gamez ME, Patel SH, Mcgee LA, Sio TT, Mcdonald M, Phan J, et al. A Systematic Review on Re-Irradiation With Charged Particle Beam Therapy in the Management of Locally Recurrent Skull Base and Head and Neck Tumors. Int J Part Ther (2021) 8:131-54. doi: 10.14338/IJPT-20-00064.1

18. Moeller BJ, Yordy JS, Williams MD, Giri U, Raju U, Molkentine DP, et al. DNA Repair Biomarker Profiling of Head and Neck Cancer: Ku80 Expression Predicts Locoregional Failure and Death Following Radiotherapy. Clin Cancer Res (2011) 17:2035-43. doi: 10.1158/1078-0432.CCR-10-2641

19. Sano D, Xie TX, Ow TJ, Zhao M, Pickering CR, Zhou G, et al. Disruptive TP53 Mutation is Associated With Aggressive Disease Characteristics in an Orthotopic Murine Model of Oral Tongue Cancer. Clin Cancer Res (2011) 17:6658-70. doi: 10.1158/1078-0432.CCR-11-0046

20. Skinner HD, Sandulache VC, Ow TJ, Meyn RE, Yordy JS, Beadle BM, et al. TP53 Disruptive Mutations Lead to Head and Neck Cancer Treatment Failure Through Inhibition of Radiation-Induced Senescence. Clin Cancer Res (2012) 18:290-300. doi: 10.1158/1078-0432.CCR-11-2260

21. Eschrich SA, Pramana J, Zhang H, Zhao H, Boulware D, Lee JH, et al. A Gene Expression Model of Intrinsic Tumor Radiosensitivity: Prediction of Response and Prognosis After Chemoradiation. Int J Radiat Oncol Biol Phys (2009) 75:489-96. doi: 10.1016/j.ijrobp.2009.06.014

22. Zhao M, Sano D, Pickering CR, Jasser SA, Henderson YC, Clayman GL, et al. Assembly and Initial Characterization of a Panel of 85 Genomically Validated Cell Lines From Diverse Head and Neck Tumor Sites. Clin Cancer Res (2011) 17:7248-64. doi: 10.1158/1078-0432.CCR-11-0690

23. Ang KK, Trotti A, Brown BW, Garden AS, Foote RL, Morrison WH, et al. Randomized Trial Addressing Risk Features and Time Factors of Surgery Plus Radiotherapy in Advanced Head-and-Neck Cancer. Int J Radiat Oncol Biol Phys (2001) 51:571-8. doi: 10.1016/S0360-3016(01)01690-X

24. Mirandola A, Molinelli S, Vilches Freixas G, Mairani A, Gallio E, Panizza D, et al. Dosimetric Commissioning and Quality Assurance of Scanned Ion Beams at the Italian National Center for Oncological Hadrontherapy. Med Phys (2015) 42:5287-300. doi: 10.1118/1.4928397

25. Durante M, Paganetti H, Pompos A, Kry SF, Wu X, Grosshans DR. Report of a National Cancer Institute Special Panel: Characterization of the Physical Parameters of Particle Beams for Biological Research. Med Phys (2019) 46: e37-52. doi: 10.1002/mp.13324

26. Lind BK, Persson LM, Edgren MR, Hedlof I, Brahme A. RepairableConditionally Repairable Damage Model Based on Dual Poisson Processes. Radiat Res (2003) 160:366-75. doi: 10.1667/0033-7587(2003)160[0366: RRDMBO]2.0.CO;2

27. Ding LH, Xie Y, Park S, Xiao G, Story MD. Enhanced Identification and Biological Validation of Differential Gene Expression via Illumina WholeGenome Expression Arrays Through the Use of the Model-Based Background Correction Methodology. Nucleic Acids Res (2008) 36:e58. doi: 10.1093/nar/ gkn234

28. Xie Y, Wang X, Story M. Statistical Methods of Background Correction for Illumina BeadArray Data. Bioinformatics (2009) 25:751-7. doi: 10.1093/ bioinformatics/btp040

29. Chen M, Xie Y, Story M. An Exponential-Gamma Convolution Model for Background Correction of Illumina BeadArray Data. Commun Stat Theory Methods (2011) 40:3055-69. doi: 10.1080/03610921003797753

30. Antonovic L, Dasu A, Furusawa Y, Toma-Dasu I. Relative Clinical Effectiveness of Carbon Ion Radiotherapy: Theoretical Modelling for H\&N Tumours. J Radiat Res (2015) 56:639-45. doi: 10.1093/jrr/rrv016

31. Johansen J, Bentzen SM, Overgaard J, Overgaard M. Relationship Between the In Vitro Radiosensitivity of Skin Fibroblasts and the Expression of Subcutaneous Fibrosis, Telangiectasia, and Skin Erythema After Radiotherapy. Radiother Oncol (1996) 40:101-9. doi: 10.1016/0167-8140(96)01777-X

32. Giri U, Ashorn CL, Ramdas L, Stivers DN, Coombes K, El-Naggar AK, et al. Molecular Signatures Associated With Clinical Outcome in Patients With
High-Risk Head-and-Neck Squamous Cell Carcinoma Treated by Surgery and Radiation. Int J Radiat Oncol Biol Phys (2006) 64:670-7. doi: 10.1016/ j.ijrobp.2005.08.032

33. Keck MK, Zuo Z, Khattri A, Stricker TP, Brown CD, Imanguli M, et al. Integrative Analysis of Head and Neck Cancer Identifies Two Biologically Distinct HPV and Three non-HPV Subtypes. Clin Cancer Res (2015) 21:87081. doi: 10.1158/1078-0432.CCR-14-2481

34. Gao J, Hu J, Guan X, Yang J, Hu W, Kong L, et al. Salvage Carbon-Ion Radiation Therapy For Locoregionally Recurrent Head and Neck Malignancies. Sci Rep (2019) 9:4259. doi: 10.1038/s41598-019-39241-y

35. Held T, Windisch P, Akbaba S, Lang K, El Shafie R, Bernhardt D, et al. Carbon Ion Reirradiation for Recurrent Head and Neck Cancer: A Single-Institutional Experience. Int J Radiat Oncol Biol Phys (2019) 105:803-11. doi: 10.1016/ j.ijrobp.2019.07.021

36. Pulte D, Brenner H. Changes in Survival in Head and Neck Cancers in the Late 20th and Early 21st Century: A Period Analysis. Oncologist (2010) 15:994-1001. doi: 10.1634/theoncologist.2009-0289

37. Duprez F, Madani I, Bonte K, Boterberg T, Vakaet L, Derie C, et al. IntensityModulated Radiotherapy for Recurrent and Second Primary Head and Neck Cancer in Previously Irradiated Territory. Radiother Oncol (2009) 93:563-9. doi: 10.1016/j.radonc.2009.10.012

38. Giro C, Berger B, Bolke E, Ciernik IF, Duprez F, Locati L, et al. High Rate of Severe Radiation Dermatitis During Radiation Therapy With Concurrent Cetuximab in Head and Neck Cancer: Results of a Survey in EORTC Institutes. Radiother Oncol (2009) 90:166-71. doi: 10.1016/j.radonc. 2008.09.007

39. Werbrouck J, De Ruyck K, Duprez F, Veldeman L, Claes K, Van Eijkeren M, et al. Acute Normal Tissue Reactions in Head-and-Neck Cancer Patients Treated With IMRT: Influence of Dose and Association With Genetic Polymorphisms in DNA DSB Repair Genes. Int J Radiat Oncol Biol Phys (2009) 73:1187-95. doi: 10.1016/j.ijrobp.2008.08.073

40. Riaz N, Hong JC, Sherman EJ, Morris L, Fury M, Ganly I, et al. A Nomogram to Predict Loco-Regional Control After Re-Irradiation for Head and Neck Cancer. Radiother Oncol (2014) 111:382-7. doi: 10.1016/ j.radonc.2014.06.003

41. Takiar V, Garden AS, Ma D, Morrison WH, Edson M, Zafereo ME, et al. Reirradiation of Head and Neck Cancers With Intensity Modulated Radiation Therapy: Outcomes and Analyses. Int J Radiat Oncol Biol Phys (2016) 95:1117-31. doi: 10.1016/j.ijrobp.2016.03.015

42. Hoebers F, Heemsbergen W, Moor S, Lopez M, Klop M, Tesselaar M, et al. Reirradiation for Head-and-Neck Cancer: Delicate Balance Between Effectiveness and Toxicity. Int J Radiat Oncol Biol Phys (2011) 81:e111-118. doi: 10.1016/j.ijrobp.2011.01.004

43. Mcdonald MW, Zolali-Meybodi O, Lehnert SJ, Estabrook NC, Liu Y, CohenGadol AA, et al. Reirradiation of Recurrent and Second Primary Head and Neck Cancer With Proton Therapy. Int J Radiat Oncol Biol Phys (2016) 96:808-19. doi: 10.1016/j.ijrobp.2016.07.037

44. Phan J, Sio TT, Nguyen TP, Takiar V, Gunn GB, Garden AS, et al. Reirradiation of Head and Neck Cancers With Proton Therapy: Outcomes and Analyses. Int J Radiat Oncol Biol Phys (2016) 96:30-41. doi: 10.1016/ j.ijrobp.2016.03.053

45. Romesser PB, Cahlon O, Scher ED, Hug EB, Sine K, Deselm C, et al. Proton Beam Reirradiation for Recurrent Head and Neck Cancer: Multi-Institutional Report on Feasibility and Early Outcomes. Int J Radiat Oncol Biol Phys (2016) 95:386-95. doi: 10.1016/j.ijrobp.2016.02.036

46. Lee EK, Song KA, Chae JH, Kim KM, Kim SH, Kang MS. GAGE12 Mediates Human Gastric Carcinoma Growth and Metastasis. Int J Cancer (2015) 136:2284-92. doi: 10.1002/ijc.29286

47. Gjerstorff MF, Terp MG, Hansen MB, Ditzel HJ. The Role of GAGE Cancer/ Testis Antigen in Metastasis: The Jury is Still Out. BMC Cancer (2016) 16:7. doi: 10.1186/s12885-015-1998-y

48. Nin DS, Wujanto C, Tan TZ, Lim D, Damen JMA, Wu KY, et al. GAGE Mediates Radio Resistance in Cervical Cancers via the Regulation of Chromatin Accessibility. Cell Rep (2021) 36:109621. doi: 10.1016/ j.celrep.2021.109621

49. Chen F, Long Q, Fu D, Zhu D, Ji Y, Han L, et al. Targeting SPINK1 in the Damaged Tumour Microenvironment Alleviates Therapeutic Resistance. Nat Commun (2018) 9:4315. doi: 10.1038/s41467-018-06860-4 
50. Xu L, Lu C, Huang Y, Zhou J, Wang X, Liu C, et al. SPINK1 Promotes Cell Growth and Metastasis of Lung Adenocarcinoma and Acts as a Novel Prognostic Biomarker. BMB Rep (2018) 51:648-53. doi: 10.5483/ BMBRep.2018.51.12.205

51. Fladeby C, Gupta SN, Barois N, Lorenzo PI, Simpson JC, Saatcioglu F, et al. Human PARM-1 Is a Novel Mucin-Like, Androgen-Regulated Gene Exhibiting Proliferative Effects in Prostate Cancer Cells. Int J Cancer (2008) 122:1229-35. doi: 10.1002/ijc.23185

52. Charfi C, Levros LCJr., Edouard E, Rassart E. Characterization and Identification of PARM-1 as a New Potential Oncogene. Mol Cancer (2013) 12:84. doi: 10.1186/1476-4598-12-84

53. Capes-Davis A, Theodosopoulos G, Atkin I, Drexler HG, Kohara A, Macleod RA, et al. Check Your Cultures! A List of Cross-Contaminated or Misidentified Cell Lines. Int J Cancer (2010) 127:1-8. doi: 10.1002/ijc.25242

54. Matsumoto Y, Matsuura T, Wada M, Egashira Y, Nishio T, Furusawa Y. Enhanced Radiobiological Effects at the Distal End of a Clinical Proton Beam: In Vitro Study. J Radiat Res (2014) 55:816-22. doi: 10.1093/jrr/rrt230

55. Kanai T, Endo M, Minohara S, Miyahara N, Koyama-Ito H, Tomura H, et al. Biophysical Characteristics of HIMAC Clinical Irradiation System for HeavyIon Radiation Therapy. Int J Radiat Oncol Biol Phys (1999) 44:201-10. doi: 10.1016/S0360-3016(98)00544-6
56. Toma-Dasu I, Dasu A, Brahme A. Dose Prescription and Optimisation Based on Tumour Hypoxia. Acta Oncol (2009) 48:1181-92. doi: 10.3109/ 02841860903188643

Conflict of Interest: The authors declare that the research was conducted in the absence of any commercial or financial relationships that could be construed as a potential conflict of interest.

Publisher's Note: All claims expressed in this article are solely those of the authors and do not necessarily represent those of their affiliated organizations, or those of the publisher, the editors and the reviewers. Any product that may be evaluated in this article, or claim that may be made by its manufacturer, is not guaranteed or endorsed by the publisher.

Copyright (C) 2022 Ding, Sishc, Polsdofer, Yordy, Facoetti, Ciocca, Saha, Pompos, Davis and Story. This is an open-access article distributed under the terms of the Creative Commons Attribution License (CC BY). The use, distribution or reproduction in other forums is permitted, provided the original author(s) and the copyright owner(s) are credited and that the original publication in this journal is cited, in accordance with accepted academic practice. No use, distribution or reproduction is permitted which does not comply with these terms. 